\title{
RELEVÂNCIA DA ASSISTÊNCIA FARMACÊUTICA NO CONTROLE DA PRESSÃO ARTERIAL SISTÊMICA
}

\author{
RELEVANCE OF PHARMACEUTICAL ASSISTANCE IN THE CONTROL OF \\ SYSTEMIC BLOOD PRESSURE
}

\author{
Hercules Venâncio Santos Almeida ${ }^{1}$ \\ Nadiane Carla Oliveira Alves ${ }^{2}$ \\ Taiane Neri de Almeira ${ }^{3}$ \\ Tiago Miranda Lago Requião ${ }^{4}$ \\ Juliana Azevedo de Paixão ${ }^{5}$
}

RESUMO: A Hipertensão Arterial Sistêmica consiste em um problema de saúde pública de difícil controle, em que fatores extrínsecos e intrínsecos podem consistir em elementos preponderantes para a dificuldade no controle da patologia. O presente estudo tem como objetivos: realizar levantamento bibliográfico sobre a importância da Assistência Farmacêutica no controle da pressão arterial sistêmica . Trata-se de uma revisão integrativa de literatura, de natureza qualitativa, descritiva e exploratória. Para a pesquisa foi realizada uma revisão da literatura científica. Os dados foram coletados a partir da Biblioteca Virtual em Saúde (BVS). Foram incluídos artigos delimitados como, estudos populacionais, estudos clínicos randomizado que estejam publicados no idioma português, disponíveis na forma gratuita e íntegra, publicados entre os anos de 2011 a 202I. Foram excluídos artigos, dissertações, teses que não atendam ao objetivo, questão norteadora e os que estiverem duplicados nas bases de dados. $O$ estudo evidenciou que entre os fatores que mais dificultam o controle da HAS, estão relacionados a hábitos culturais e estilo de vida, relação entre a equipe e o paciente acometido pela patologia, o contexto familiar em que o indivíduo doente esta inserido, além da complexidade dos esquemas terapêuticos.

Palavras-chave: Hipertensão arterial sistêmica. Atenção Farmacêutica. Idosos .

ABSTRACT: Systemic Arterial Hypertension is a public health problem that is difficult to control, in which extrinsic and intrinsic factors can be preponderant elements for the difficulty in controlling the pathology. The present study aims to: carry out a bibliographic survey on the importance of Pharmaceutical Assistance in the control of systemic blood pressure. It is a narrative literature review, qualitative, descriptive and exploratory. For the research, a review of the scientific literature was carried out. Data were collected from the Virtual Health Library (VHL). Articles delimited as population studies, randomized clinical trials that are published in

\footnotetext{
${ }^{1}$ Graduando em farmácia . UNIFACS

${ }^{2}$ Graduando em farmácia . UNIFACS

3 Graduando em farmácia. UNIFACS

${ }^{4}$ Graduando em farmácia. UNIFACS

5 Farmacêutica . UFBA.
} 
Portuguese, available free of charge and in their entirety, published between the years 2011 to 2021, were included. Articles, dissertations, theses that do not meet the objective, guiding question were excluded. and those that are duplicated in the databases. The study showed that among the factors that most hinder the control of $\mathrm{SAH}$, they are related to cultural habits and lifestyle, the relationship between the team and the patient affected by the pathology, the family context in that the sick individual is inserted, in addition to the complexity of the therapeutic schemes.

Keywords: Systemic arterial hypertension. Pharmaceutical Care. Elderly.

\section{INTRODUÇÃO}

No Brasil, desde 1998, ocorreu a inserção da Assistência Farmacêutica na agenda do governo federal, resultando na criação da Política Nacional de Medicamentos (PNM), permitindo desde então a inserção de medidas como, adoção de uma lista de medicamentos essenciais; regulamentação sanitária de medicamentos; reformulação da assistência farmacêutica; promoção do uso racional de medicamentos; desenvolvimento científico e tecnológico; promoção da produção; garantia de segurança, eficácia e qualidade dos medicamentos e desenvolvimento e capacitação de recursos humanos (BERMUDEZ et al.,2018).

A Assistência Farmacêutica é reconhecida e regulamentada no Brasil pelo Ministério da Saúde sob Portaria no 2.982, de 26 de novembro de 2009 que estabelece as normas de execução e financiamento da Assistência Farmacêutica na atenção básica em saúde. Mas somente em 2010 houve a criação do Departamento de Assistência Farmacêutica (DASF) (KOPPITTE; CAMILO,20II).

Como instrumento aliado da Assistência Farmacêutica tem-se uma relação de medicamentos padronizados, conhecida como Relação Nacional de Medicamentos Essenciais (RENAME). Esta relação é elaborada através da Medicina Baseada em Evidências, com fundamento para orientação da prescrição e do abastecimento da rede do Sistema Único de Saúde (SUS), com vista no aperfeiçoamento de questões administrativas, redução de custos e principalmente no Uso Racional de Fármacos (YAMAUTI et al.,2017).

Como um instrumento norteador a RENAME facilita as ações de planejamento, aquisição de medicamentos e de organização da Assistência Farmacêutica no âmbito do SUS, sendo assim, as equipes de saúde, principalmente 
os profissionais que possuem a autonomia da prescrição, devem seguir a RENAME e o Formulário Terapêutico Nacional, para poder realizar a escolha adequada da melhor terapêutica a cada paciente (SANTANA; CATANHEIDE, 2015).

Em relação a população e usuários do SUS, a RENAME configura-se em um compromisso da garantia à disponibilização de medicamentos eleitos de acordo a padrões de ordem técnico-científicos e de acordo com as prioridades de saúde da população (OLIVEIRA; ASSIS; BARBONI, 20I2).

No planejamento da Assistência Farmacêutica, estão as doenças crônicas que causam uma maior influência na vida população brasileira, que vem trazendo um impacto nas taxas de mortalidade. Em relação às doenças crônicas que causam influencia na vida das pessoas, há uma atenção especial para a Hipertensão Arterial Sistêmica ou HAS, que é uma condição clínica multifatorial, que se caracteriza por níveis elevados e sustentados da pressão arterial com associação de transformações na função dos órgãos afetados (rins, vasos sanguíneos, coração e encéfalo), assim como as alterações metabólicas, modificações no volume do líquido circulante e resistência vascular periférica resultando em riscos cardiovasculares (LIM et al.,2013).

A manifestação e a gravidade da HAS são influenciadas por fatores como: idade, fatores socioeconômicos, excesso do consumo de sódio, obesidade, sedentarismo, etilismo. A hipertensão arterial sistêmica por ser uma morbidade de caráter crônico é vista por Malachias et al. (2016) como um problema que acarreta de elevado custo social e de grande impacto no perfil de morbimortalidade da população brasileira, traduzindo-se um desafio para o sistema público de saúde.

Diante do desafio supracitado, o Ministério da Saúde, desenvolveu com a colaboração do Conselho Nacional de Secretários de Saúde, Conselho Nacional de Secretários Municipais de Saúde, o Plano de Reorganização da Atenção à Hipertensão Arterial e ao Diabetes Mellitus, com este plano pretende-se que ocorra um aumento da prevenção, diagnóstico, tratamento e controle da hipertensão arterial e do Diabetes Mellitus, por intermédio da reorganização da Rede Básica dos Serviços de Saúde/SUS, visando a resolutividade e qualidade no atendimento, acarretando na redução do número de internações, a diminuição da demanda no pronto atendimento, e os gastos com tratamento de complicações, aposentadorias precoces e a mortalidade 
cardiovascular, com a consequente melhoria da qualidade de vida da população(PRATES et al.,2020).

A Assistência farmacêutica favorece a interação com o paciente, de modo que esclarece dúvidas relacionadas a administração do medicamento, além disso, o profissional também fica comprometido em realizar o acompanhamento farmacológico dos pacientes, com foco em resultados positivos do tratamento através de resolução de problemas que possa emergir. No acompanhamento estão inclusos objetivos importantes, como a responsabilização do paciente em utilizar o medicamento prescrito pelo médico em esquemas posologicos corretamente para que seja seguro e eficaz resultando em efeito terapêutico desejado, bem como a certificação de que as reações adversas ao uso do medicamento sejam mínimas e que se aparecerem sejam solucionadas de modo imediato pelo profissional farmacêutico (PERES; PEREIRA, 2015).

Deste modo, a escolha do tema desse estudo justifica-se pela possibildade de socialização de informações acerca da Assistencia Farmacêutica, contribuindo com a difusão do tema para a comunidade acadêmica, sociedade e profissionais que assistem pacientes com hipertensão arterial sistêmica. Bem como, o presente trabalho possibilitará o melhor entendimento por parte dos futuros profissionais de saúde acerca do tema proposto.

Face ao exposto, a abordagem do tema deste estudo é relevante, porque faz um questionamento sobre a importância da Assistência Farmacêutica no controle da pressão arterial sistêmica, visto que o profissional foca no acompanhamento farmacológico dos pacientes,assegurando o alcance de resultados positivos da terapeutica através da resolutividade de problemas que podem emergir.

Para tanto este estudo tem como objetivo :realizar levantamento bibliográfico sobre a importância da Assistência Farmacêutica no controle da pressão arterial sistêmica .

\section{METODOLOGIA}

Esse estudo é uma revisão integrativa da literatura, de natureza qualitativa, descritiva e exploratória. Para a pesquisa foi realizada uma revisão narrativa da 
literatura científica. Desse modo, os dados serão coletados a partir da Biblioteca Virtual em Saúde (BVS), utilizando-se as bases de dados Medical Literature Analysis and Retrieval System On-line (MEDLINE) e Literatura Latino-Americana e do Caribe em Ciências da Saúde (LILACS), Bases de Dados da Enfermagem (BDENF), Public Medline (PubMed), CAPES Periódicos, além da Scientific Electronic Library Online (SciELO). Para a pesquisa nas bases de dados serão utilizados os seguintes descritores: hipertensão arterial sistêmica, controle de hipertensão, tratamento medicamentoso, assistentência farmacêutica.

Como critérios de inclusão foram selecionados artigos, artigos delimitados como, estudos populacionais, estudos clínicos randomizado que estejam publicados no idioma português, disponíveis na forma gratuita e íntegra, publicados entre os anos de 2011 a 2021. Foram excluídos artigos, dissertações, teses que não atendam ao objetivo, questão norteadora e os que estiverem duplicados nas bases de dados (contabilizados apenas uma vez).

A técnica de análise de dados se processou através da leitura de todo o material selecionado na íntegra para posterior categorização temática. Seguindo essa linha, a cada leitura dos materiais utilizados, elaborou-se- um resumo próprio, a partir da reflexão e interpretação do entendimento sobre o estudo, ou a cada informação considerada de grande relevância. Em consonância com a Lei 9610/98 que aborda a relação do plágio em obras literárias com o registro de autoria para proteger e preservar os direitos dos autores.

\section{RESULTADOS E DISCUSSÃO}

Um total de 136 artigos foi identificado nas bases de dados, sendo que 22 duplicatas foram removidas, resultando em II4 estudos submetidos à triagem de título e resumo. Esta triagem inicial removeu 25 artigos que não atenderam aos critérios de seleção. Posteriormente, com a leitura completa dos 89 artigos elegíveis, os mesmos atenderam aos critérios estabelecidos do trabalho, resultando em 37 artigos selecionados para este estudo. O fluxograma na Figura or mostra o processo de seleção dos estudos. 
Figura I - Processo de seleção dos estudos.

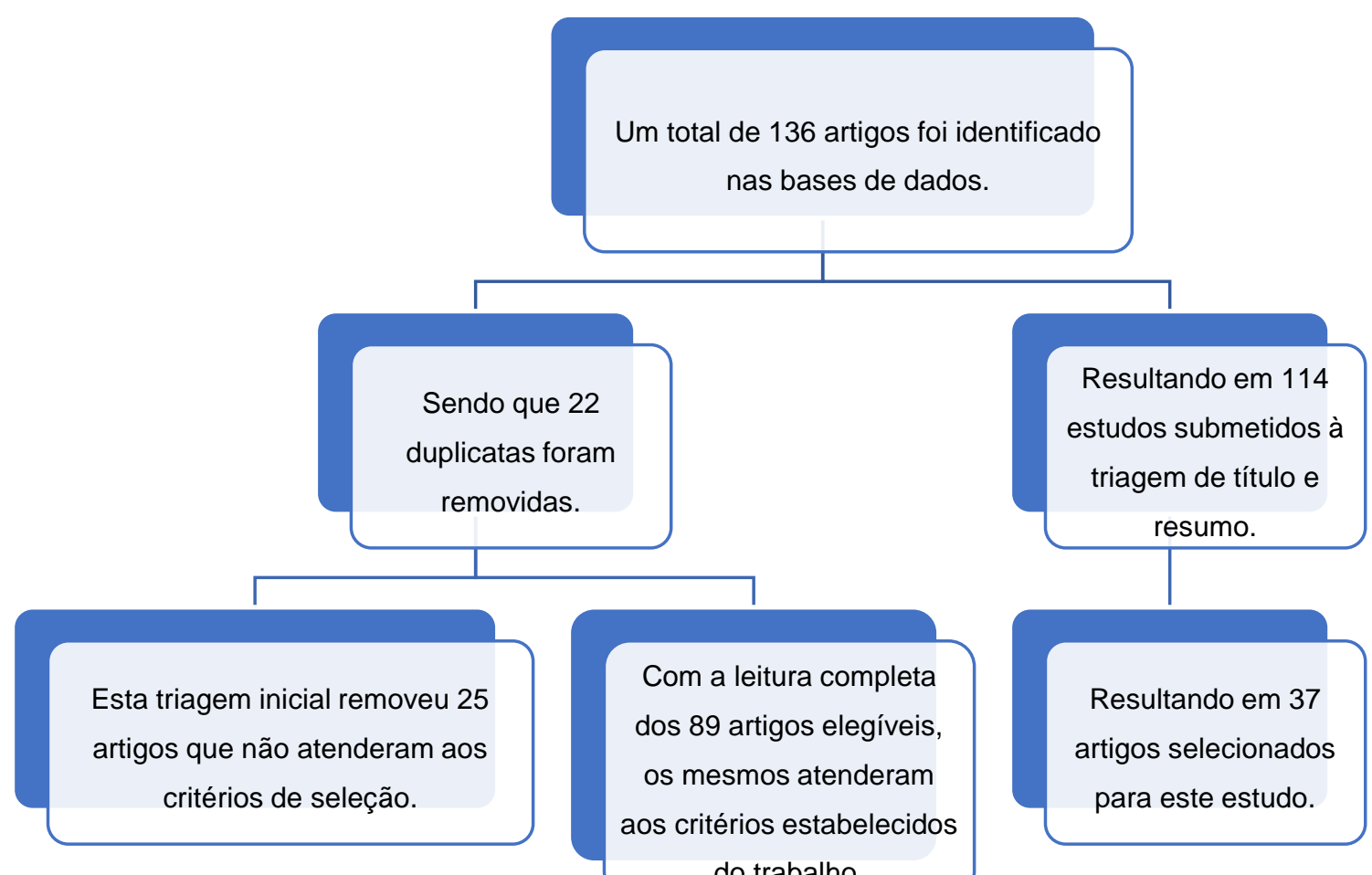

Fonte: dados da pesquisa (2021)

Nos estudos foi mostrado que a hipertensão arterial (HA) é uma doença crônica não transmissível (DCNT) definida por níveis pressóricos, em que os benefícios do tratamento (não medicamentoso e/ ou medicamentoso) superam os riscos. Trata-se de uma condição multifatorial, que depende de fatores genéticos/ epigenéticos, ambientais e sociais, caracterizada por elevação persistente da pressão arterial (PA), ou seja, PA sistólica (PAS) maior ou igual a $140 \mathrm{mmHg}$ e/ou PA diastólica (PAD) maior ou igual a $90 \mathrm{mmHg}$, medida com a técnica correta, em pelo menos duas ocasiões diferentes, na ausência de medicação anti-hipertensiva. É aconselhável, quando possível, a validação de tais medidas por meio de avaliação da $\mathrm{PA}$ fora do consultório por meio da Monitorização Ambulatorial da Pressão Arterial (MAPA), da Monitorização Residencial da Pressão Arterial (MRPA) ou da Automedida da Pressão Arterial (AMPA)( LOBO et al.,2017).

Foi discutido que por se tratar de condição frequentemente assintomática, a HA costuma evoluir com alterações estruturais e/ou funcionais em órgãos-alvo, como coração, cérebro, rins e vasos. Ela é o principal fator de risco modificável com 
associação independente, linear e contínua para doenças cardiovasculares (DCV), doença renal crônica (DRC) e morte prematura. Associa-se a fatores de risco metabólicos para as doenças dos sistemas cardiocirculatório e renal, como dislipidemia, obesidade abdominal, intolerância à glicose, e diabetes melito (DM)( OIGMAN; NEVES; GISMONDI,2015).

Alguns autores mostraram que existe um impacto significativo nos custos médicos e socioeconômicos, decorrentes das complicações nos órgãos-alvo, fatais e não fatais, como: coração: doença arterial coronária (DAC), insuficiência cardíaca (IC), fibrilação atrial (FA) e morte súbita; cérebro: acidente vascular encefálico (AVE) isquêmico (AVEI) ou hemorrágico (AVEH), demência; rins: DRC que pode evoluir para necessidade de terapia dialítica; e sistema arterial: doença arterial obstrutiva periférica (DAOP)( MONTEIRO et al.,2020).

A HA tem alta prevalência e é um dos principais fatores de risco para as DCV e renais, apresentando determinantes genéticos, ambientais e sociais combinados. Mostra-se de fácil diagnóstico e seu tratamento é eficaz utilizando-se um arsenal terapêutico diversificado, bastante eficiente e com poucos efeitos adversos. Mesmo assim, seu controle em todo o mundo é pífio, porque se trata de doença frequentemente assintomática, o que dificulta a adesão aos cuidados(FALCÃO et al.,2018).

A equação final torna o desafio do tratamento muito elevado, e a prevenção continua a ser a melhor opção em termos de custo-benefício. A abordagem adequada dos fatores de risco para o desenvolvimento da HA deve ser o grande foco do SUS. Nesse quesito, há vários pontos que merecem destaque. Muitos se confundem ou se somam ao tratamento não medicamentoso(SILVA; BRANDÃO; LIMA,2017).

\section{HAS : TRATAMENTO MEDICAMENTOSO}

Uma das primeiras medidas que são tomadas em relação ao paciente hipertenso ou que tenha tendência a se tornar hipertenso, são as mudanças no seu estilo de vida, pois reduzem a PA, bem como a mortalidade cardiovascular. As principais recomendações não medicamentosas para prevenção da HAS são: alimentação saudável, consumo controlado de sódio e is de álcool, ingestão de potássio e combate ao sedentarismo e ao tabagismo, além de controlar o peso, corporal. Essa é uma 
maneira de contribuir positivamente para a saúde do paciente, e quando é seguido fielmente, a necessidade de ingestão de medicamentos nem sempre se faz necessária . Porém, é válido ressaltar que mesmo que seja necessário o uso de medicamentos, a terapia não-medicamentosa constitui uma importante aliada no tratamento de todos os pacientes hipertensos. $\mathrm{O}$ tratamento medicamentoso da $\mathrm{HA}$ visa, em última análise, a redução da morbimortalidade CV(ARAÚJO; ARAÚJO,2020).

Foi mostrada evidências científicas através de estudos clínicos de desfechos que mostram benefícios do tratamento realizado com o uso de diuréticos ,Betabloqueadores, inibidores da enzima conversora de angiotensina, bloqueadores dos receptores ATI da angiotensina II . Deve ser ressaltado que a maioria desses estudos utilizou medicamentos em associação. Com base nas informações disponíveis, a proteção observada não depende do tipo de fármaco empregado, mas fundamentalmente da redução da PA(FERREIRA et al.,2017).

O tratamento com medicamentos está indicado para os indivíduos com PA estágio r e risco CV baixo e moderado, quando as medidas não farmacológicas não surtirem efeito após um período inicial de pelo menos 90 dias. Em situações especiais, onde o acesso e/ou retorno a assistência médica seja difícil, poderá ser considerado o emprego inicial de medicação anti-hipertensiva, mesmo para esse grupo de pacientes. Para aqueles em estágio I e alto risco CV ou DCV estabelecida, o uso de medicamentos deverá ser iniciado de imediato. Da mesma maneira, nos casos de HA estágio 2 e 3, independentemente do risco $\mathrm{CV}$, o tratamento medicamentoso deverá ser iniciado de imediato. Para os indivíduos com pré-hipertensão, a utilização de medicamentos poderá ser uma opção, levando-se em consideração o risco CV e/ou a presença de DCV. Em pacientes de 60 a 79 anos, com PAS $\geq 140 \mathrm{mmHg}$ e naqueles com $\geq 80$ anos com PAS $\geq 160 \mathrm{mmHg}$, o início da terapia medicamentosa deverá ser mais precoce(LOBO et al.,2017).

O princípio básico do manejo farmacológico é a combinação de medicamentos anti-hipertensivos que tenham ação sobre grande parte dos mecanismos fisiopatológicos de elevação da pressão tais quais : expansão do volume intravascular, a ativação simpática e do sistema Renina-Angiotensina-Aldosterona e a resistência vascular periférica aumentada. $\mathrm{O}$ melhor tratamento triplo deve incluir um 
diurético (DIU) tiazídico, um bloqueador do SRAA (inibidor da ECA ou bloqueador dos receptores ATI da angiotensina (BRA) II) e um bloqueador dos canais de cálcio (BCC) di-hidropiridínico de longa duração, em doses plenas e toleradas a intervalos adequados. $\mathrm{Na}$ presença de doença arterial coronariana, insuficiência cardíaca ou taquiarritmias, um betabloqueador (BB) deve substituir o BCC no esquema terapêutico inicial com três medicações(ISSA et al.,202I) .

No que concerne aos Diuréticos, sabe-se que o mecanismo da ação antihipertensiva dos destes medicamentos está relacionado aos efeitos natriuréticos, com a redução do volume circulante e do volume extracelular e que depois de quatro a seis semanas, o volume circulante normaliza-se, o que pormove a redução da resistência vascular periférica (MALACHIAS , 2016)

Sobre os Bloqueadores dos Canais de Cálcio esta é uma calsse de medicamentos que bloqueia os canais de cálcio na membrana das células musculares lisas das arteríolas, reduzindo a disponibilidade de cálcio no interior das células dificultando a contração muscular e, desta forma há uma diminuição a resistência vascular periférica por vasodilatação(MACHADO; PEPE; ARINELLI,2017).

Os Inibidores da Enzima Conversora da Angiotensina (IECA) consistem em medicamentos anti-hipertensivos que possui como mecanismo de ação a inibição da enzima conversora de angiotensina I, responsável a um só tempo pela transformação de angiotensina I em angiotensina II (vasoconstritora) e pela redução da degradação da bradicinina (vasodilatadora)(BRITO; DIAS; OLIVEIRA,2020).

Os betabloqueadores possuem ações farmacológicas complexas. No entanto, promovem a diminuição inicial do débito cardíaco e da secreção de renina, com a readaptação dos barorreceptores e diminuição das catecolaminas nas sinapses nervosas (BARROSO et al.,202I).

Os Simpatolíticos de Ação Central possuem coomo mecanismo de ação do estímulo dos receptores alfa-2 que estão envolvidos nos mecanismos simpatoinibitórios. Quanto aos efeitos bem definidos essa classe são: diminuição da atividade simpática e do reflexo dos barorreceptores, o que contribui para a bradicardia relativa e a hipotensão notada na posição ortostática; discreta diminuição na RVP e no débito cardíaco; redução nos níveis plasmáticos de renina; e retenção de 
fluidos(MACHADO et al.,202I).

Os alfabloqueadores consistem em medicamentos que agem como antagonistas competitivos dos receptores alfa-ı pós-sinápticos, reduzindo a resistencia vascular perifericas sem mudanças no débito cardíaco. Promovem maior redução pressórica quando na posição ortostática e na taquicardia reflexa(ISSA et al.,202I).

No que diz respeito aos Inibidores Diretos da Renina, estes promovem a inibição direta da ação da renina com a consequente diminuição da formação de angiotensina II.Outras ações podem contribuir para a redução da PA e a proteção tissular, como redução da atividade plasmática de renina,bloqueio de receptor celular próprio de renina/pró-reninas 46 e diminuição da síntese intracelular de angiotensina II( MALACHIAS et al.,2017).

\section{FATORES QUE INTERFEREM NO CONTROLE DA HIPERTENSÃO ARTERIAL}

As doenças crônicas compõem o conjunto de condições crônicas. Normalmente estão relacionadas a causas múltiplas, são caracterizadas por início gradual, de prognóstico incerto, com longa ou indefinida duração. Apresentam curso clínico que muda ao longo do tempo, com possíveis períodos de agudização, podendo gerar incapacidades. Demandam intervenções com o utilização de tecnologias leves, leveduras e duras, associadas a mudanças de estilo de vida, em um processo de cuidado contínuo que nem sempre leva à cura(BRASIL,2013).

As doenças cardiovasculares (DCV) constituem atualmente a principal causa de mortalidade e a terceira de morbidade a nível mundial, dentre elas, a hipertensão arterial sistêmica (HAS)é uma das mais importantes, apesar de ter havido grandes avanços no desenvolvimento de novos fármacos anti-hipertensivos a partir da década de 5o, capazes de reduzir a pressão arterial(FERREIRA et al., 2015).

Existem elementos relacionados à terapêutica que pode interferir no controle da hipertensão arterial. Nesta direção o desconhecimento acerca as comorbidades associadas à HAS, que pode consistir em um fator que colabore para o descontrole da hipertensão (MIRON, 20I6). Os estudos discutiram quais fatores mais inteferem no controle da HAS. O grafico I, evidencia quais são esses fatores .Os fatores 
Vulnerabilidade socioeconômica, obesidade e falta de apoio familiar foram os elementos maus citados pelos autores.

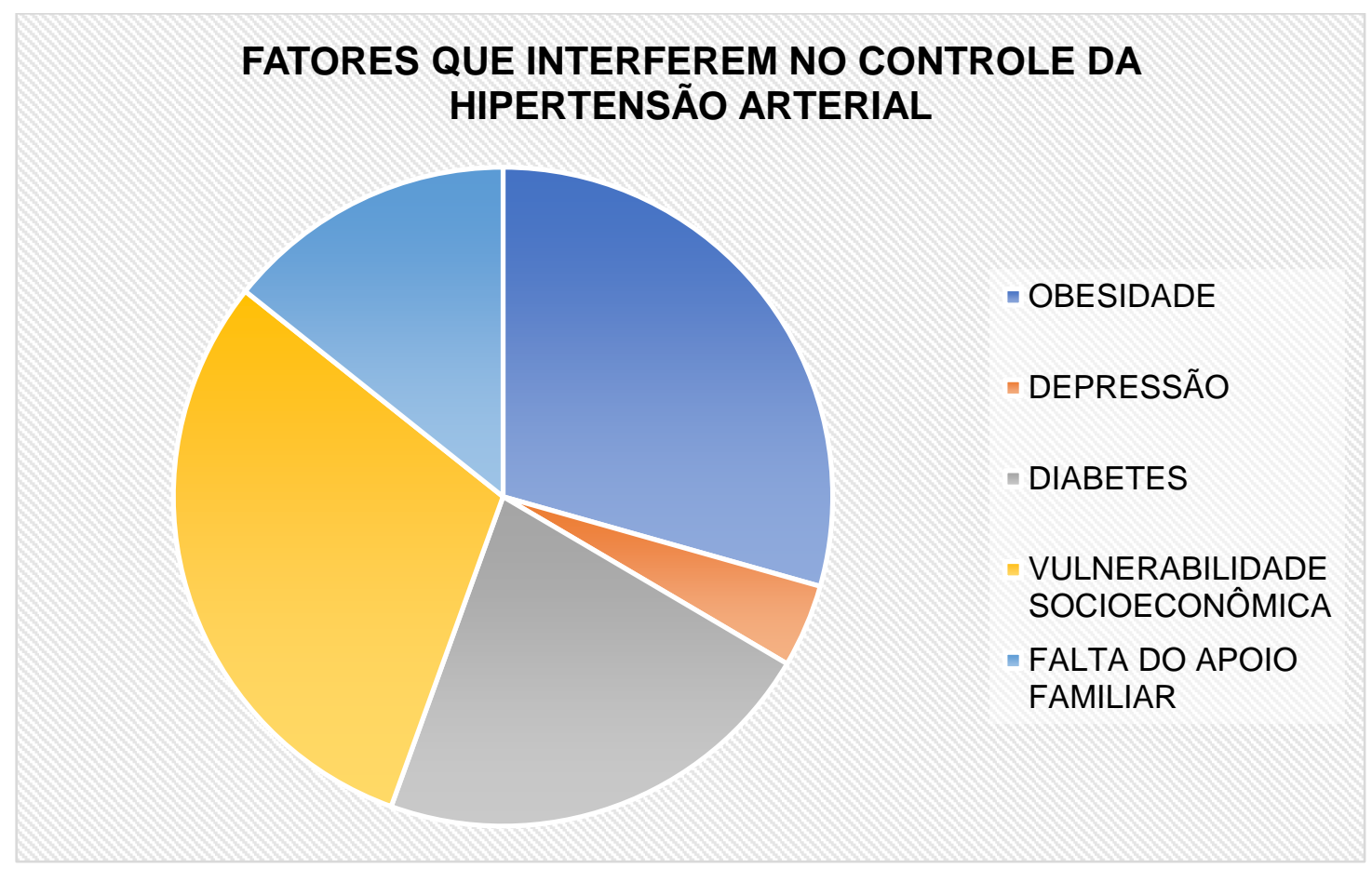

Fonte : Dados da pesquisa.

A obesidade por vezes não é considerada pelos pacientes hipertensos, como uma patologia relacionada à HAS, ou seja, as pessoas não consideram e desconhecem que a obesidade seja um elemento de risco para a instalação da hipertensão que deve ser tratada, favorecendo o controle da hipertensão (SOUZA et al., 2014).

Entretanto, é necessário que haja transformações nos hábitos de estilo de vida que incluam uma dieta mais equilibrada e a pratica regularmente de exercícios físicos, com a finalidade de controlar o peso do corpo. Contudo, é oportuno destacar, nem sempre os pacientes são sensibilizados de maneira adequada sobre a importância da adoção de uma alimentação livre de sódio, realização de atividades físicas, sendo assim, a mudança no estilo de vida fica prejudicada pela falta de uma correta orientação, se configurando em mais um fator que prejudica o controle da HAS (MACHADO; PIRES; LOBÃO, 2012).

Além disso, existem outras comorbidades que causam interferência no controle 
efetivo da HAS. A depressão pode colaborar para a não aderência a terapêutica da hipertensão arterial, além disso, alguns anti-hipertensivos, como por exemplo: a alfametildopa, a clonidina e betabloqueadores de ação central, podem provocar quadros de depressão, e os diuréticos tiazídicos podem elevar os níveis séricos de lítio (DIAS et al., 2016).

A prevalência de hipertensão arterial em paciente portadores de diabetes é cerca de duas vezes maior, em relação dos pacientes que não à possui, onde na diabetes tipo I está relacionada nefropatia diabética, sendo necessário o controle da pressão arterial com vistas a promover o retardo da perda de função renal, já no diabetes tipo 2, a HAS está ligada a outros fatores de risco cardiovasculares, como a dislipidemia, obesidade, hipertrofia ventricular esquerda e hiperinsulinemia, sendo assim, o controle ineficaz dos índices glicêmico, colabora para a elevação da PA (BORBA, 2013).

No que diz respeito a hipertensão no período gestacional, toda gestante que apresente HAS, deverá ser acompanhada quinzenalmente, depois da vigésima semana, e semanalmente, depois da trigésima semana de gestação, vale destacar que a Organização Mundial da Saúde, preconiza que o tratamento deve ser iniciado quando a pressão arterial diastólica ultrapassar $95 \mathrm{mmHg}$, contudo, nem todos os medicamentos podem ser utilizados no contexto da gravidez, como por exemplo, os Inibidores da ECA, que podem provocar um agravamento da isquemia uterina e provocar insuficiência renal no feto, e antagonistas do receptor da angiotensina II que também é um medicamento contraindicado para gestantes (MELO et al., 2015).

Os pacientes podem apresentar dificuldade em aceitar a HAS, devido a limitações, decorrente da condição crônica. O que irá influenciar na vida pessoal, sua relação familiar e comunitária, porque terão que mudar seus hábitos de vida, assumir uma rotina rigorosa que envolve o controle do peso; a realizar dieta alimentar; reduzir o consumo de sal; reduzir o consumo de bebidas alcoólicas; participarem de grupos terapêuticos; elimina o consumo de tabaco e a realizarem atividade física. Além de utilizarem medicamentos para o controle da PA. As mudanças no estilo de vida não se iniciam de imediato, mais aos poucos com o tempo, o que levará as pessoas a repensarem nos seus planos para o futuro (PERES et al., 2015).

Outro fator para a deficiência do controle da Hipertensão é a vulnerabilidade 
socioeconômica, acredita-se que questões relativas as condições socioeconômicas do paciente hipertenso tem um protagonismo importante no descontrole da HAS, já causam interferência sobre as situações de saúde, como por exemplo a acessibilidade aos serviços de saúde, no nível de conhecimento, e entendimento acerca da patologia. Sendo assim quanto menor for às condições socioeconômicas da pessoa acometida pela HAS, maior o nível de desconhecimento acerca da doença (TEIXEIRA et al., 20I6).

O déficit na formação acadêmica limita o conhecimento sobre a Hipertensão e sua terapia, onde sua prevalência é inversamente proporcional, quanto a formação acadêmica e renda, ou seja, quanto maior o nível de instrução e condição financeira, menor é a incidência de HAS, devido o acesso e aos cuidados com a saúde, visto que algumas ações realizadas pelo hipertenso, podem ser cruciais para o efetivo controle da hipertensão (TAVARES et al., 2016).

Outra limitação para o controle da HAS é a falta do apoio familiar, visto que o (FAVARO et al., 20r6). tratamento demanda como já dito anteriormente, transformações no estilo de vida, deste modo, exige uma participação de todos que fazem parte da rede social do paciente. Vale comentar que os hipertensos com uma rede familiar fragilizada, estão mais propensos a não aderirem ao tratamento proposto para o controle da patologia.

\section{ASSISTÊNCIA FARMACÊUTICA E A HIPERTENSÃO ARTERIAL}

O farmacêutico é um profissional do campo de saúde que também atua de modo a prevenir que as pessoas desenvolvam a Hipertensão Arterial, vale destacar que o fazer deste profissional também contribui para a qualidade de vida das pessoas que já possuem a patologia. No que concerne a Atenção Farmacêutica destinada a parcela da população que têm hipertensão a RDC 44/o9 preconiza que sejam realizadas a aferição da pressão arterial em farmácias e drogarias, além do acompanhamento da terapia medicamentosa (ALANO; CORREA; GALATO,2012).

Neste sentido, Alano; Correa; Galato (2012) discorre que o profissional deve se comprometer em construir procedimentos operacionais padrão, levando em conta a subjetividade de cada individuo que será acompanhado, com estes procedimentos o farmacêutico encontra bases para prestar uma atenção farmacêutica com qualidade. 
É importante lembrar que o acompanhamento da terapia medicamentosa pelos profissionais farmacêuticos de pacientes hipertensos precisa ser feito com muito comprometimento com vistas a garantir a qualidade de vida destas pessoas, além de evitar problemas relacionados aos medicamentos como já foi citado, o exemplo anteriormente, as interações medicamentosas. Um modelo de sucesso para o acompanhamento de pacientes hipertensos é um desenvolvido na Espanha, denominado de Dáder(LIMA et al.,2016) .

Lima et al.(2016) explica que ele é norteado pela terapia medicamentosa da pessoa hipertensa, além dos problemas ligados aos fármacos que o paciente está em uso. $\mathrm{O}$ autor cita ainda que a efetividade do modelo no acompanhamento da terapia do hipertenso é alguns fatores são levados em conta, são eles: a interferência da hipertensão no corpo da pessoa hipertensa, as medições da pressão arterial com vistas a prospectar a segurança, efetividade e necessidade da terapia medicamentosa, o uso concomitante de outros fármacos e quais as possibilidades de interação medicamentosa .

Mafra e colaboradores (2012), lembram que a utilização correta de fármacos colabora para melhor terapia medicamentosa, em contrapartida, o uso irracional provoca danos à saúde. Neste sentido, o uso de vários medicamentos pode provocar efeitos significativos para os indivíduos e, no grupo de idosos, esses efeitos são mais sérios, e podem ser nocivos, pois podem provocar manifestações no metabolismo. Isto porque, com o avançar da idade, há um aumento da gordura no corpo, com isso há um decréscimo de massa magra, o que pode comprometer o metabolismo, ocasionando dificuldades na eliminação de metabólitos, provocando uma aglutinação de compostos tóxicos, deste modo, há um aumento da ocorrência de eventos adversos.

Desta maneira, Mendonça (2012), indica que a assistência farmacêutica, é capaz de reduzir prejuízos e assegurar a qualidade e a eficácia do tratamento medicamentoso. A falta de adesão terapêutica medicamentosa é uma problemática frequente em todas as faixas etárias, entretanto, como os idosos usam uma quantidade maior de fármacos a falta de adesão cresce proporcionalmente ao número de drogas usadas.

Ferreira e colaboradores (2015), entre os problemas mais prevalentes na 
utilização de fármacos no grupo idosos, estão a escolha errada da droga, erro na recepção do medicamento, administração inadequada, amplificada pelos episódios de esquecimento, doses sub-terapêuticas,superdosagens,eventoss adversos, interações medicamentosas e automedicação.

Sendo assim, Silva et al. (2015), descrevem que é crucial que os profissionais percebam os problemas relacionados ao uso de fármacos na população de idosos, já que essas pessoas são mais vulneráveis aos eventos adversos provocados por medicamentos e as interações medicamentosas.

Lima; Nascimento e Grassi (2016) explicam que a atenção farmacêutica, engloba dispensação de medicamentos, e, sobretudo, oferece orientação para tomada de decisões acerca da utilização de medicamentos pelos idosos. Na tomada de decisão, estão inclusos, o não uso de certos medicamentos, bem como opiniões acerca da eleição do tratamento medicamentoso.

A assistência farmacêutica no contexto da hipertensão arterial sistêmica tem uma grande relevância. O farmacêutico que atua alinhado com a Atenção Farmacêutica, entende a importância do acompanhamento da terapia farmacológica, para identificação de problemas relacionados aos medicamentos, desta forma, garantem uma melhor qualidade de vida para os hipertensos, além da diminuição da necessidade das pessoas serem internadas, reduzindo os gastos para os sistemas de saúde e minimizando os índices de mortalidades associados aos problemas de saúde acarretados pela elevação da pressão arterial sistêmica(ARAÚJO; ARAÚJO,2020).

\section{CONSIDERAÇÕES FINAIS}

Com este estudo pode-se evidenciar que a hipertensão arterial sistêmica consiste em uma doença com um início silencioso, além de representar um fator de risco para as doenças de natureza cardiovascular, onde a prevenção da HAS e seu manejo, demanda do comprometimento de uma equipe multidisciplinar que irá promover ações educativas que tratem das complicações e da mudança no estilo de vida e na correta adesão ao tratamento medicamentoso.É importante ressaltar que, para minimizar os fatores que dificultam o controle de hipertensão arterial, é necessário que haja um trabalho que envolva o próprio paciente, a sua família, bem 
como a atenção dos profissionais de saúde, onde os portadores devem conhecer a doença e sua forma de controlá-la, bem como seus fatores de risco; ter o apoio de familiares para controle da doença, mudanças no estilo de vida e hábitos alimentares.

\section{REFERÊNCIAS}

ALANO, Graziela Modolon; CORREA, Taís dos Santos; GALATO, Dayani. Indicadores do Serviço de Atenção Farmacêutica (SAF) da Universidade do Sul de Santa Catarina. Ciênc. saúde coletiva, Rio de Janeiro , v. 17, n. 3, p. 757764, Mar. 2012.

ARAÚJO, Thadeu Rocha; ARAÚJO, Pollyana Rocha. Assistência do farmacêutico em pacientes com hipertensão. Brazilian Journal of Development, v. 6, n. 4, p. 178o6I7820, 2020.

BARROSO, Weimar Kunz Sebba et al. Diretrizes Brasileiras de Hipertensão Arterial-2020. Arquivos Brasileiros de Cardiologia, v. I16, n. 3, p. 516-658, 2021.

BERMUDEZ, Jorge Antonio Zepeda et al. Assistência Farmacêutica nos 30 anos do SUS na perspectiva da integralidade. Ciência \& Saúde Coletiva, v. 23, p. 1937-1949, 2018.

BORBA, Anna Karla de Oliveira Tito et al. Adesão à terapêutica medicamentosa em idosos diabéticos. Revista da Rede de Enfermagem do Nordeste, Fortaleza, v. I4, n. 2, p.394-404, fev. 2013.

BRASIL. MINISTÉRIO DA SAÚDE. Diretrizes para o cuidado das pessoas com doenças crônicas nas redes de atenção à saúde e nas linhas de cuidado prioritárias. 2013. Disponível em https://bvsms.saude.gov.br/bvs/publicacoes/diretrizes\%2o_cuidado_pessoas\%2o_do encas_cronicas.pdf 
BRITO, Veronica Perius; DIAS, Fellipe Leonardo Torres; OLIVEIRA, Stefan. Hipertensão arterial sistêmica, uso de bloqueadores dos receptores de angiotensina II e inibidores da enzima conversora da angiotensina e COVID-I9: Uma revisão sistemática. InterAmerican Journal of Medicine and Health, v. 3, 2020.

DIAS, Ernandes Gonçalves et al. Lifestyle and hindering factors in controlling hypertension/Estilo de vida e fatores dificultadores no controle da hipertensão/Estilo de vida y factores que complica el control de la hipertensión. Revista de Enfermagem da UFPI, v. 4, n. 3, p. 24-9, 2016.

FALCÃO, Aline et al. Estilo de vida e adesão ao tratamento de hipertensão arterial sistêmica em homens idosos. Revista Brasileira em Promoção da Saúde, v. 3I, n. 2, p. I-IO, 2018.

FAVARO, Danielli Teixeira Lima et al. Impact of educational group strategy to improve clinical and glycemic parameters in individuals with diabetes and hypertension. Investigación y Educación En Enfermería, Colombia, v. 24, n. 2, p.314321, 2016.

FERREIRA, Synara Cirelle Holanda et al. A Atenção Farmacêutica ao paciente portador de hipertensão arterial sistêmica. Mostra Científica da Farmácia, v. 3, n. I, 2017.

FERREIRA, Vinicius Lins et al. Pharmacotherapeutic monitoring of hypertensive patients in pharmacy school-UFPB. Visão Acadêmica, v. I6, n. I, 2015.

ISSA, Aurora Felice Castro et al. Diretrizes Brasileiras de Hipertensão Arterial-2020. Arq Bras Cardiol, v. 116, n. 3, p. 516-658, 2021.

KOPPITTE, Luciane; CAMILO, Elineide. Assistência Farmacêutica em um Serviço de Atenção Primária à Saúde. Tempus Actas de Saúde Coletiva, v. 4, n. 3, p. ág. 43-5I, 
2011.

LIM, Stephen S. et al. A comparative risk assessment of burden of disease and injury attributable to 67 risk factors and risk factor clusters in 21 regions, 1990-2010: a systematic analysis for the Global Burden of Disease Study 2010. The Lancet, v. 380, n. 9859, p. 2224-226o, 2013.

LIMA, Tiago Aparecido Maschio de et al . Análise de potenciais interações medicamentosas e reações adversas a anti-inflamatórios não esteroides em idosos. Rev. bras. geriatr. gerontol., Rio de Janeiro, v. 19, n. 3, p. 533-544, June 2016

LOBO, Larissa Aline Carneiro et al. Tendência temporal da prevalência de hipertensão arterial sistêmica no Brasil. Cadernos de Saúde Pública, v. 33, 2017.

MACHADO CESAR, Luiz Antonio; PEPE, Camila; ARINELlI, Roberta. Custoefetividade de trimetazidina no tratamento de angina estável em pacientes diabéticos não respondedores a betabloqueadores, nitratos e bloqueadores do canal de cálcio. JBES: Brazilian Journal of Health Economics/Jornal Brasileiro de Economia da Saúde, v. 9, n. 2, 2017.

MACHADO, Lara Coqui et al. Critérios de escolha de fármacos anti-hipertensivos em adultos. Brazilian Journal of Health Review, v. 4, n. 2, p. 6756-6775, 2021.

MACHADO, Mariana Carvalho; PIRES, Cláudia Geovana da Silva; LOBAO, William Mendes. Concepções dos hipertensos sobre os fatores de risco para a doença. Ciênc. saúde coletiva, Rio de Janeiro, v. 17, n. 5, p. 1357 1363, May 2012.

MAFRA, Paola Di Bernardi et al. Uso de medicamentos na perspectiva da racionalidade e segurança: um estudo de base populacional. Revista Brasileira de Hipertensão, v. 238, n. 7, p. 47-70, 2012 
MALACHIAS, Marcus Vinícius Bolívar et al. $7^{\text {a }}$ Diretriz Brasileira de Hipertensão Arterial: Capítulo I-Conceituação, Epidemiologia e Prevenção Primária. Arquivos Brasileiros de Cardiologia, v. 107, n. 3, p. I-6, 2016

MELO, Wyara Ferreira et al. A hipertensão gestacional e o risco de pré-eclampsia: revisão bibliográfica. Revista Brasileira de Educação e Saúde, v. 5, n. 3, p. 07-II, 2015

MENDONÇA, Rodrigo Eduardo Braga de. O cuidado farmacêutico na prevenção da intoxicação medicamentos. Revista Brasileira de Hipertensão, v. I2, n. 2, p. 23-52, 2012.

MIRON, Irene Caroline Matos Nardy. Plano de intervenção para impactar a não adesão ao tratamento da hipertensão arterial no PSF de Arantina-Minas Gerais. Revista Brasileira de Hipertensão, v. 23, n. 2, p. 47-51, 2016. 2016.

MONTEIRO, Ariane Alice Fernandes et al. Estudo sobre a adesão ao tratamento de hipertensão arterial sistêmica na UBSF de Três Poços. Brazilian Journal of Health Review, v. 3, n. I, p. 1289-1305, 2020.

OIGMAN, Wille; NEVES, Mario Fritsch; GISMONDI, Ronaldo Altenburg Odebrecht Curi. Hipertensão arterial sistêmica. RBM rev. bras. med, 2015.

OLIVEIRA, Luciane Cristina Feltrin de; ASSIS, Marluce Maria Araújo; BARBONI, André René. Assistência farmacêutica no Sistema Único de Saúde: da Política Nacional de Medicamentos à atenção básica à saúde. Ciência \& Saúde Coletiva, v. I5, p. 356I-3567, 2012.

PERES, Heverton Alves; CASTRO, Sergio Henrique Rafaine de.Tratamento farmacológico e interações medicamentosas em pacientes com aneurisma da aorta abdominal. Infarma-Ciências Farmacêuticas, v. 27, n. 2, p. 135-141, 2015.

PRATES, Elton Junio Sady et al. Características clínicas de clientes com hipertensão 
arterial e diabetes mellitus. Rev. enferm. UFPE on line, p. [I-10], 2020.

SANTANA, Rafael Santos; CATANHEIDE, Izamara Damasceno. Relação Nacional de Medicamentos: uma construção permanente. Cadernos de Saúde Pública, v. 3I, n. 3, p. 647-647, 2015 .

SILVA, Andreia Soares; BRANDÃO, Elineuda de Sousa Parente; LIMA, Liene Ribeiro. Assistência farmacêutica ao paciente idoso portador de doenças crônicas e arterial sistêmica. Mostra Científica da Farmácia, v. 3, n. I, 2017.

SILVA, Lorena Flor da Rosa Santos et al. Fatores associados à adesão ao tratamento anti-hipertensivo por idosos na atenção primária. Revista de Ciências Farmacêuticas Básica e Aplicada, v. 35, n. 2, p. 271-278, 2015.

SOUZA, Clarita Silva de et al . Blood Pressure Control in Hypertensive Patients in the "Hiperdia Program": A Territory-Based Study. Arq. Bras. Cardiol., São Paulo, v. 102, n. 6, p. 571-578. 2014 .

TAVARES, Darlene Mara dos Santos et al. Quality of life and accession to the pharmacological treatment among elderly hypertensive. Revista Brasileira de Enfermagem, v. 69, n. I, p. I34-I4I, 2016.

TEIXEIRA, Juliana de Fátima et al. Hypertensives' Knowledge About High-Sodium Foods and Their Behavior. Arquivos Brasileiros de Cardiologia, v. I06, n. 5, p. 404-410, 2016.

YAMAUTI, Sueli Miyuki et al. Essencialidade e racionalidade da relação nacional de medicamentos essenciais do Brasil. Ciência \& Saúde Coletiva, v. 22, p. 975-986, 2017. 